\title{
Optical properties of dust aggregates in the disk of Beta Pictoris
}

\author{
R. Nakamura \\ Information Processing Center, Kobe University, Rokko-dai 1-1, Nada, Kobe, Japan
}

(Received December 8, 1997; Revised January 7, 1998; Accepted January 25, 1998)

\begin{abstract}
Dust particles in the disk of Beta Pictoris $(\beta$ Pic) is modeled by fluffy aggregates consisting of slightly modified interstellar grains. We calculate the optical properties by Discrete Dipole Approximation with a1-term method. It is found that the characteristic size of an aggregate is defined by the radius of volume-equivalent sphere. The wavelength dependence of the scattering efficiency in visible becomes flat when the volume-equivalent sphere exceeds $1 \mu \mathrm{m}$ irrespective of the monomer size, while the infrared silicate feature is present even for aggregates larger than $10 \mu \mathrm{m}$. Therefore, our model can account for the coexistence of the neutral scattering and silicate feature in the $\beta$ Pic disk without detailed tuning of the dust size distribution. Due to the enhancement of geometrical cross section, aggregates generally show higher scattering and absorption efficiencies in visible compared with the volume-equivalent sphere. In contrast, the absorption efficiency for an aggregate is comparable to that for the volume-equivalent sphere when the size is smaller than wavelength. These properties are also consistent with the observed superheat and high albedo of the dust particles in the $\beta$ Pic disk.
\end{abstract}

\section{Introduction}

Beta Pictoris is the best studied Vega-type star with the optically resolved circumstellar dust disk. However, the typical size of dust particles in the disk is still an open question. Several optical observations (Paresce and Burrows, 1987; Lecavelier des Etangs et al., 1993) have indicated that the color of the disk is same as the $\beta$ Pic itself within the observational uncertainties. This result has been interpreted that dust size, responsible for the scattered light, is larger than $1 \mu \mathrm{m}$. On the other hand, infrared observations suggest a smaller size less than $1 \mu \mathrm{m}$. Telesco et al. (1988) observed thermal emission from the disk at 10 and 20 microns. From the deduced color temperature much higher than blackbody, they concluded that the dust particles, which emit most of thermal infrared radiation, must be smaller than $1 \mu \mathrm{m}$. Mid infrared spectroscopy (Aitken et al., 1993; Knacke et al., 1993) also infers that submicron or micron-sized particles dominate thermal radiation from the disk because the observed silicate feature would diminish if the typical dust size exceeds several microns.

The dust particles around $1 \mu \mathrm{m}$ can produce both the silicate feature and neutral scattering. If the dust disk is not a transient phenomenon, however, this single-sized dust model has a serious difficulty. As Artymowicz (1988) has shown, dust particles around $1 \mu \mathrm{m}$ are subject to rapid ejection from the system due to the high radiation pressure forces. An alternative explanation is bimodal size distributions, in which larger component is responsible for scattered light and smaller component dominate thermal radiation. But this model requires fine tuning of the size distribution. It is unlikely that the proper size distribution is realized over the whole disk. This problem may be relevant to zodiacal cloud.

Copy right $(\mathrm{C}$ The Society of Geomagnetism and Earth, Planetary and Space Sciences (SGEPSS); The Seismological Society of Japan; The Volcanological Society of Japan; The Geodetic Society of Japan; The Japanese Society for Planetary Sciences.
Recent space-borne infrared observations of zodiacal light (Reach et al., 1996; Ootsubo et al., in this issue) imply the existence of the silicate feature around $10 \mu \mathrm{m}$, while the color is similar to the sun with a moderate amount of reddening (Matsuura et al., 1995).

It is well known that a class of interplanetary dust particles (IDPs) consists of aggregates with fluffy structure. Recently, Xing and Hanner (1997) demonstrated that such aggregates can reproduce the phase function and polarization of zodiacal light. Several authors have pointed out that the phase function and polarization of zodiacal light can successfully reproduce the visible brightness/polarization distributions in the $\beta$ Pic disk (Kalas and Jewitt, 1996; Artymowicz, 1997). In addition, the silicate feature observed in the $\beta$ Pic disk closely resembles that for IDPs (Knacke et al., 1993). Based on these striking similarities to IDPs and zodiacal cloud, we propose a new model for the dust around $\beta$ Pic, i.e., fluffy aggregates composed of interstellar dust particles. Section 2 describes the model and computational procedure. The results and discussions are presented in Section 3.

\section{Dust Models and Computational Procedure}

Our starting point is the standard model of interstellar dust, so-called MRN (Mathis et al., 1977; Draine and Lee, 1984), in which dust particles are represented by spheres with a power-law size distribution. We have modified this basic model as follows.

In its original form, MRN model allows for only silicate and carbon because it was originally developed for dust grains in diffuse clouds. In contrast, ices can be major components in the outer part of the $\beta$ Pic disk from the equilibrium temperature. However, ices are excluded from our model because recent calculations of photosputtering by hard UV (Artymowicz, 1997) has shown that the icy surface layer would be removed in a relatively short time scale. We assume 


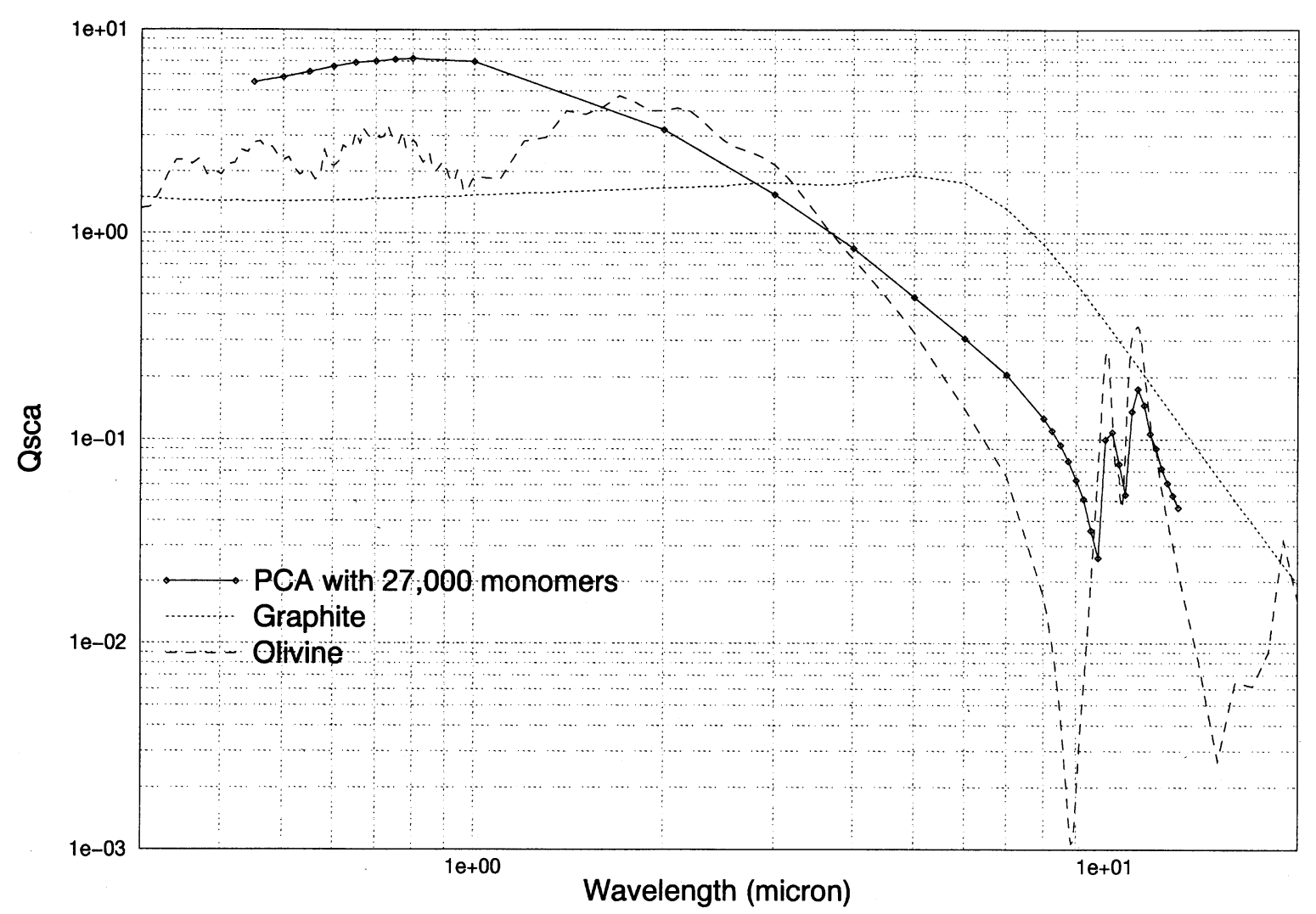

Fig. 1. The wavelength dependence of the scattering efficiency $Q_{\text {sca }}$ of PCA with 27,000 monomers of $0.03 \mu \mathrm{m}$. Also shown are the curves for the volume-equivalent sphere $(a=0.9 \mu \mathrm{m})$ composed by pure olivine and graphite.

that the carbon component is graphite as in MRN model, but the silicate component should be modified as follows. The infrared silicate feature of the $\beta$ Pic disk is different from that of interstellar dust. While the former has double peaks, indicating the presence of crystalline silicate, the latter with broad single peak is generally attributed to amorphous silicate. This change may reflect some thermal alteration processes experienced by dust particles along the evolutional path from interstellar to circumstellar environments. Therefore, we employ the optical constants of crystalline olivine (Mukai and Koike, 1990) in the following calculations instead of those for astronomical silicate provided by MRN model. Although several studies have shown that olivine can be a major component of IDPs (Sandford and Walker, 1985) and interstellar dust particles (Greenberg and Li, 1996), we do not insist that this specific composition is a unique solution. Similarly, graphite should be considered as just a example of carbonaceous absorbing materials rather than a realistic components of circumstellar dust particles.

Since the collisional or Poynting-Robertson time scales are much shorter than the expected age of $\beta$ Pic system (Artymowicz, 1997), dust particles must be replenished in the disk. Up to now, several mechanisms have been proposed to supply dust particles, such as collisional cascade (Nakano, 1988; Artymowicz, 1997), gigantic cometary swarm (Lecavelier des Etangs et al., 1996), and collisions between planetesimals and interstellar dust (Whitmire et al., 1992; Yamamoto and Mukai, 1998). In any model, dust particles are ejected as fragments from their fragile parent planetesimals. Then, we assume that they have fluffy structures like chondritic porous IDPs of cometary origin. Among several models to represent the complicated geometry of such aggregates, we choose Particle-Cluster Aggregation (PCA) in this work because of the compact structure. We refer to Mukai et al. (1992) for further detail of this model. The size of constituent particles is assumed to be identical for simplicity. It seems unlikely that aggregates with higher porosity, such as Cluster-Cluster Aggregation, survive the high velocity collisions which had yielded dust particles in the $\beta$ Pic disk. Since coagulation is presumed to mix the constituent particles uniformly in the disk, the monomers in an aggregates are randomly set as olivine or graphite with the number ratio 1:1 following MRN model.

The optical properties of PCA are calculated by Discrete Dipole Approximation (Draine, 1988). In its original form, a target with arbitrary geometry is represented by an array of dipoles. In contrast, we consider the aggregates composed of spherical monomers. Each spherical monomer is replaced by one dipole and the polarizability is derived by a1-term method (Okamoto, 1995). As demonstrated by Okamoto and $\mathrm{Xu}$ (in this issue), the cross sections for such aggregates can be computed within 10 percent errors as long as the size parameter for a monomer is smaller than unity. It means that the validity criteria depend on what properties we are interested in. For example, monomer size should be less than $0.07 \mu \mathrm{m}$ when we compare the model calcu- 


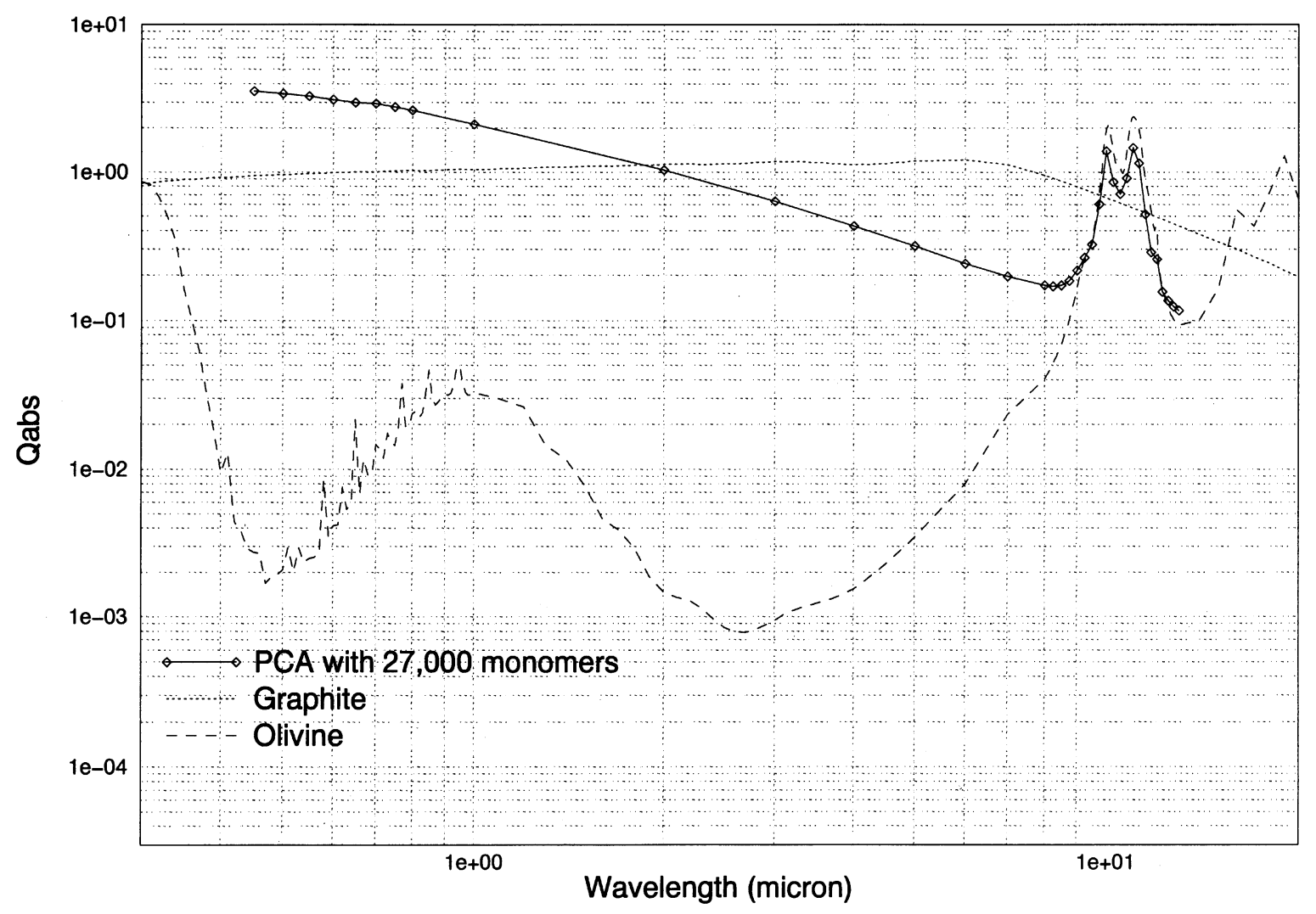

Fig. 2. Same as Fig. 1, but for absorption effciencies $Q_{\text {abs. }}$.

lations with the disk color observation in visible. On the other hand, the monomers of $1 \mu \mathrm{m}$ are allowed, considering only mid infrared absorptions. Since the a1-term method significantly reduces the memory requirement and CPU time, we can calculate the optical properties of large aggregates, whose volume-equivalent size parameters are greater than 20 , with averaging over 125 orientations.

\section{Results and Discussion}

Figure 1 shows the scattering efficiency $Q_{\text {sca }}$ from visible to mid infrared for PCA with 27,000 monomers normalized by the geometrical cross section of the volume-equivalent sphere. The radius of monomers and the volume-equivalent sphere is 0.03 and $0.9 \mu \mathrm{m}$, respectively. The curves for the volume-equivalent spheres composed by pure olivine and graphite are displayed for comparison. It should be noted that $Q_{\text {sca }}$ and $Q_{\text {abs }}$ for PCA show no resonance structures in visible. This is probably because irregularly shaped aggregates cause no definite phase shift required for interference. We found that the aggregate has larger scattering efficiency $Q_{\text {sca }}$ in visible compared to the volume-equivalent sphere. Similarly, the visible absorption efficiency $Q_{\text {abs }}$ is much higher than the volume-equivalent spheres as seen in Fig. 2, while the infrared absorption lies between those for pure olivine and graphite.

The average albedo $A$ of dust particles in the $\beta$ Pic disk are supposed to be so high $(\sim 0.5 \pm 0.2)$ (Artymowicz et al., 1989; Backman et al., 1992) that Artymowicz (1997) speculated the dust particles in the disk are extremely transparent. However, it is difficult to deplete all the absorbing materials completely and selectively from the disk. The value of $A$ is derived by the formula

$$
A=\frac{Q_{\text {sca }}}{Q_{\text {sca }}+Q_{\mathrm{abs}}}
$$

where $Q_{\text {sca }}$ and $Q_{\text {abs }}$ are determined from optical and infrared observations. Our model can yield high albedo in comparison with the volume-equivalent sphere due to the larger scattering cross sections in visible and comparable mid-infrared emission efficiency (Figs. 1 and 2). In addition, the relative increase of absorption efficiency in visible to that in mid infrared would leads to the higher temperature than the volume-equivalent sphere. This effect is also in accordance with the infrared observation addressed in the previous section (Telesco et al., 1988). Unfortunately, the quantitative estimate of the temperature cannot be made since we need cross sections at shorter wavelengths, where $\beta$ Pic is the most luminous and a1-term method cannot be applied. Xing and Hanner (1997) estimated the temperature of the aggregates in our solar system, dividing large monomers into multiple dipoles. They found that the temperature is lower than that for the single monomer, but still higher than blackbody.

Figure 3 indicates the wavelength dependence of $Q_{\text {sca }}$ in visible. Fixing the monomer radius $a_{0}$ as $0.03 \mu \mathrm{m}$, we examine the effect of the size of an aggregate, i.e., the monomer number. Meanwhile, the monomer size dependence of $Q_{\text {sca }}$ is displayed in Fig. 4, where the total number of monomers is fixed as 27,000 . We can convert the monomer size $a_{0}$ to the radius of volume-equivalent sphere $a$ simply by multiplying 


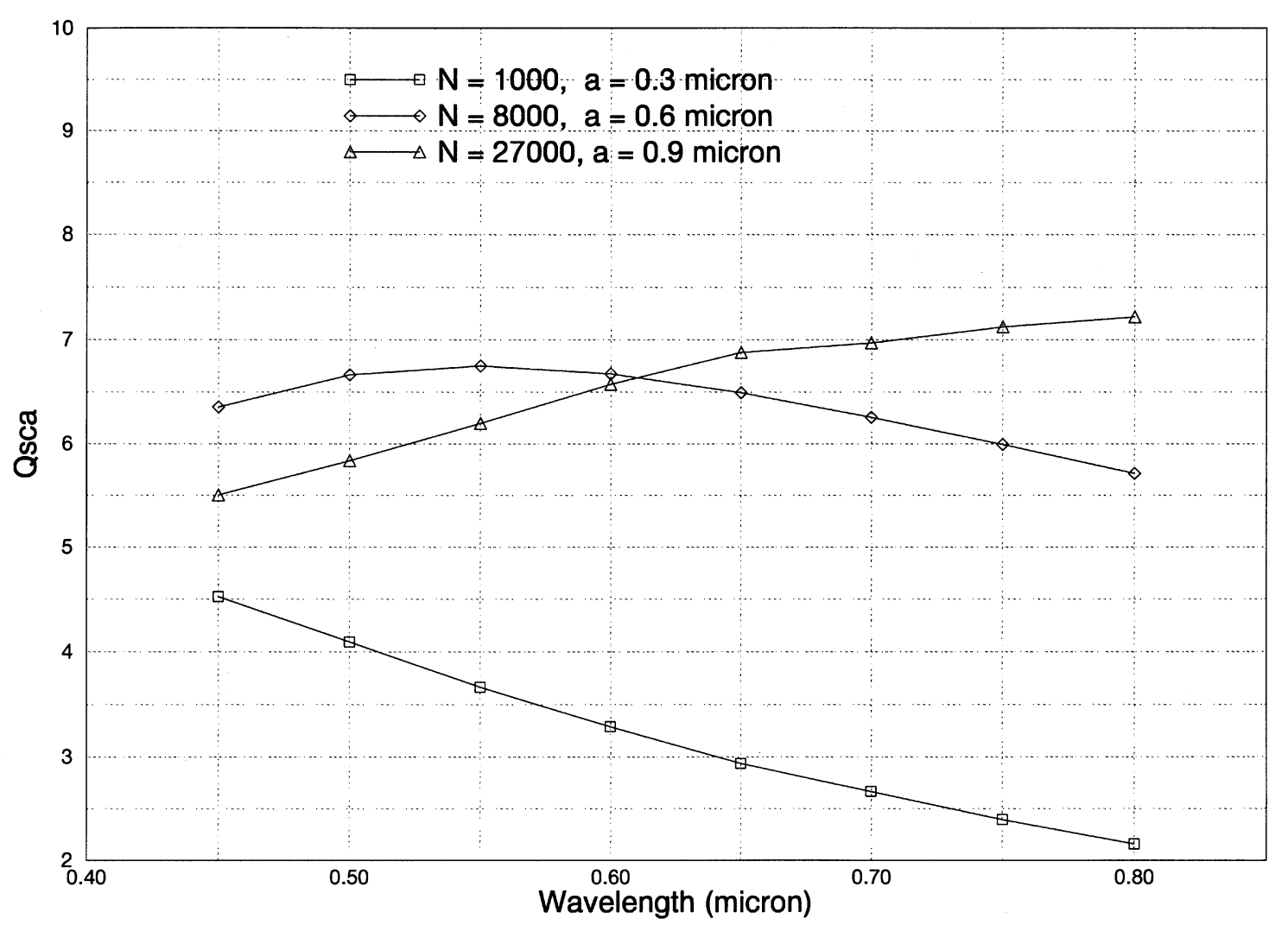

Fig. 3. The wavelength dependence of scattering efficiencies for aggregates with $0.03 \mu \mathrm{m}$ monomers. As the number $N$ varies from 1000 to 27,000 , the volume-equivalent radii $a$ increase from 0.3 to $0.9 \mu \mathrm{m}$.

$N^{1 / 3}$, where $N$ denotes the number of monomers. Then, the volume-equivalent radii $a$ are 30 times larger than the monomer size in Fig. 4. The curves on both figures seem to have a turn-over point around $X \sim 2 \pi$ independent of monomer size, where the size parameter $X$ of the aggregate is defined as $2 \pi a / \lambda$. The absolute values increase with the monomer number in Fig. 3 and decrease as the monomer size increase in Fig. 4. This trend can be understood in terms of the geometrical cross section of PCA normalized by the volume-equivalent sphere, which approaches constant in large $N$ limit (Mukai et al., 1992). The insensitivity of $Q_{\text {sca }}$ to the monomer size, as well as the correlation with geometrical cross section, can be confirmed in Fig. 5, where the monomer size dependence of $Q_{\text {sca }}$ is displayed for a fixed aggregate size of $0.6 \mu \mathrm{m}$. Since the scattering efficiency of an aggregate seems to be controlled by the geometrical cross section when $X>1$, the color of scattered light from PCA becomes neutral in visible when the volume-equivalent radius exceeds roughly $1 \mu \mathrm{m}$. Moreover, the discussion in last paragraph about the high temperature and albedo of aggregates can be true only if the volume-equivalent radius is smaller than the wavelength where the thermal emission is most effective. This may restrict the upper limit size of aggregates in our model.

Alternatively, the mid-infrared silicate feature may give the upper limit. As the total number of constituent particles is limited by our accessible computer resources, we enlarge the size of aggregates by increasing monomer size. In this case, the optical properties in visible cannot be calculated because of the large size parameter for monomers. The infrared absorption cross sections for PCA with 27,000 monomers is plotted in Fig. 6. The monomer size is set as $0.2 \mu \mathrm{m}$, near the upper end of MRN size distribution, and $0.5 \mu \mathrm{m}$. Also shown are the absorption efficiencies for the volumeequivalent spheres composed by pure olivine. In spite of the large size and graphite inclusion, the aggregates produce the clear features with double peaks. As shown in previous figures, the irregular shape of the aggregates smear out the size parameter resonance in visible. Then, why do the infrared features exist? It is worth noting that the infrared feature of $Q_{\text {abs }}$ has a different origin from that for the $Q_{\text {sca }}$ structure in visible. While the former comes from the variation of the refractive index caused by Si-O stretching band, the latter results from the size parameter resonance. Presumably, the interaction between incident radiation and $\mathrm{Si}-\mathrm{O}$ stretching band is dominated by the size of constituent particles, rather than the whole size of the aggregate.

The most serious difficulty of our model is same as that for single-sized spherical dust model, i.e., the dynamical stability. Moreover, fluffy aggregates should experience larger radiation pressure because they have larger cross sections in visible as shown in Figs. 1 and 2. The accurate calculations of the radiation pressure force on PCA, as well as the temperature, cannot be performed since the optical cross sections are required at shorter wavelengths where the a1-term method breaks down. We make only a crude estimate as fol- 


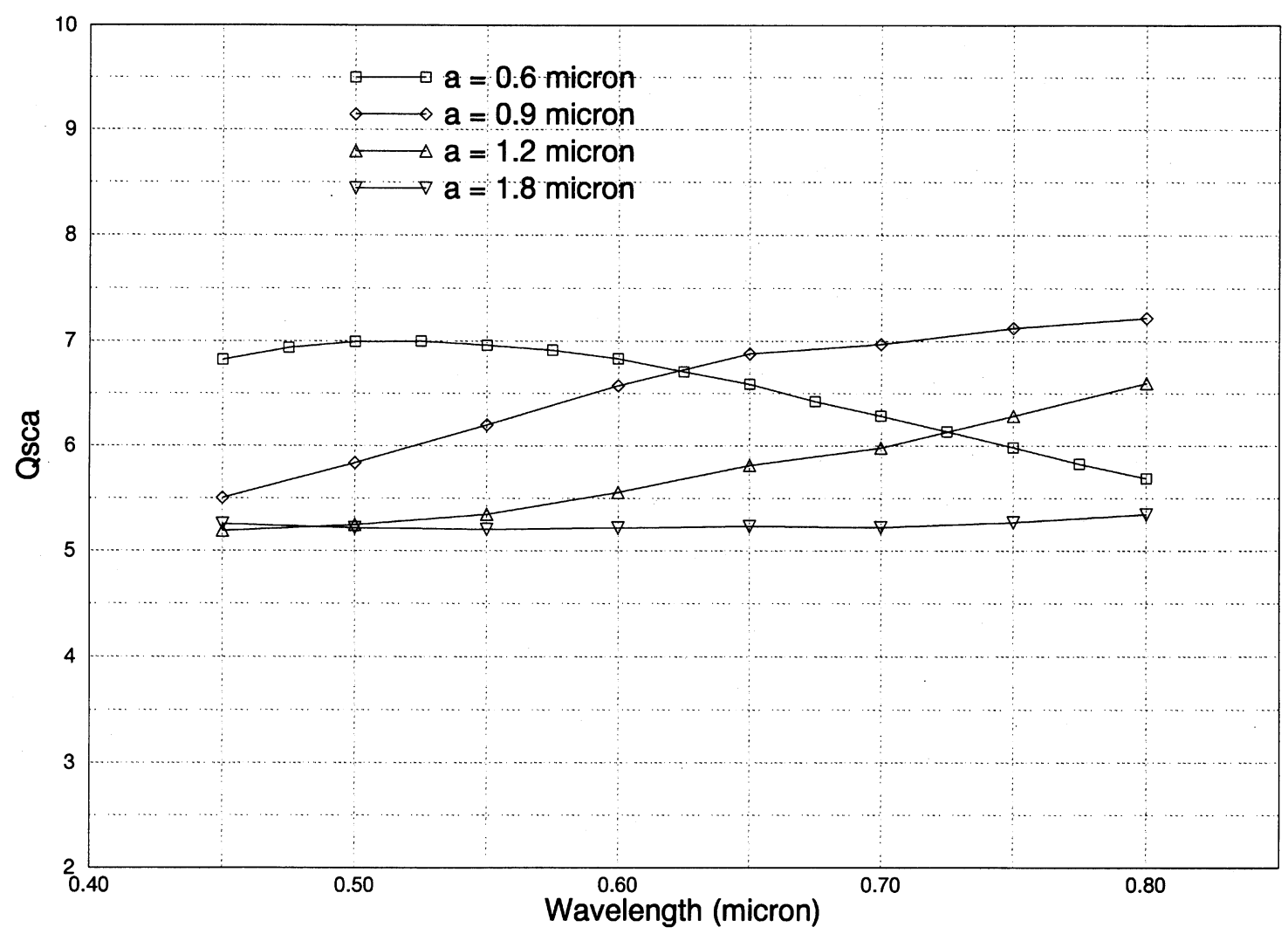

Fig. 4. Same as Fig. 3, but for a fixed monomer number $(N=27,000)$. The volume-equivalent radii $a$ of aggregates increases with the monomer size.

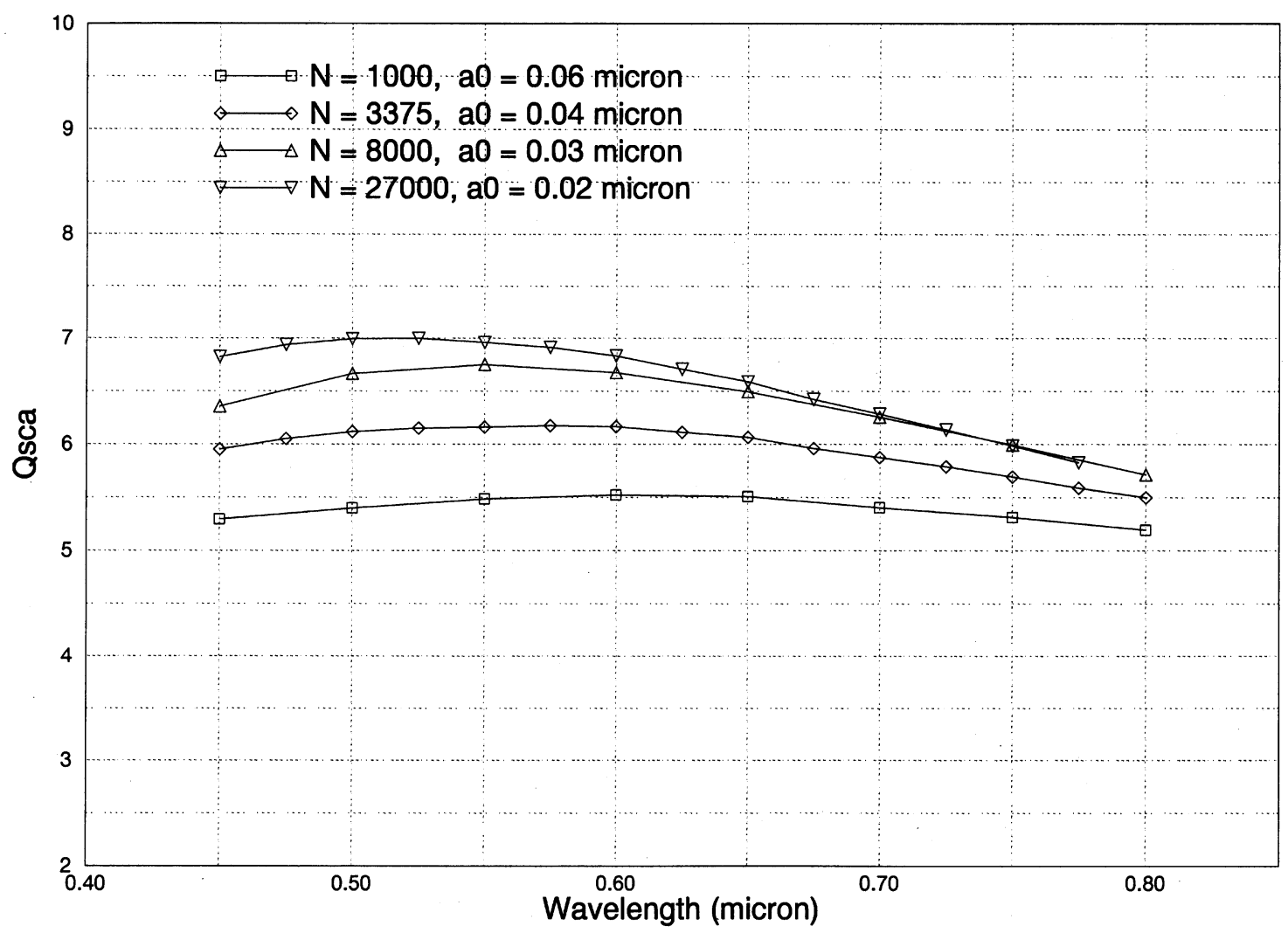

Fig. 5. Same as Fig. 3, but for a fixed aggregate size $(a=0.6 \mu \mathrm{m})$. The monomer size ranges from $0.02(N=27,000)$ to $0.06(N=1,000) \mu \mathrm{m}$. 


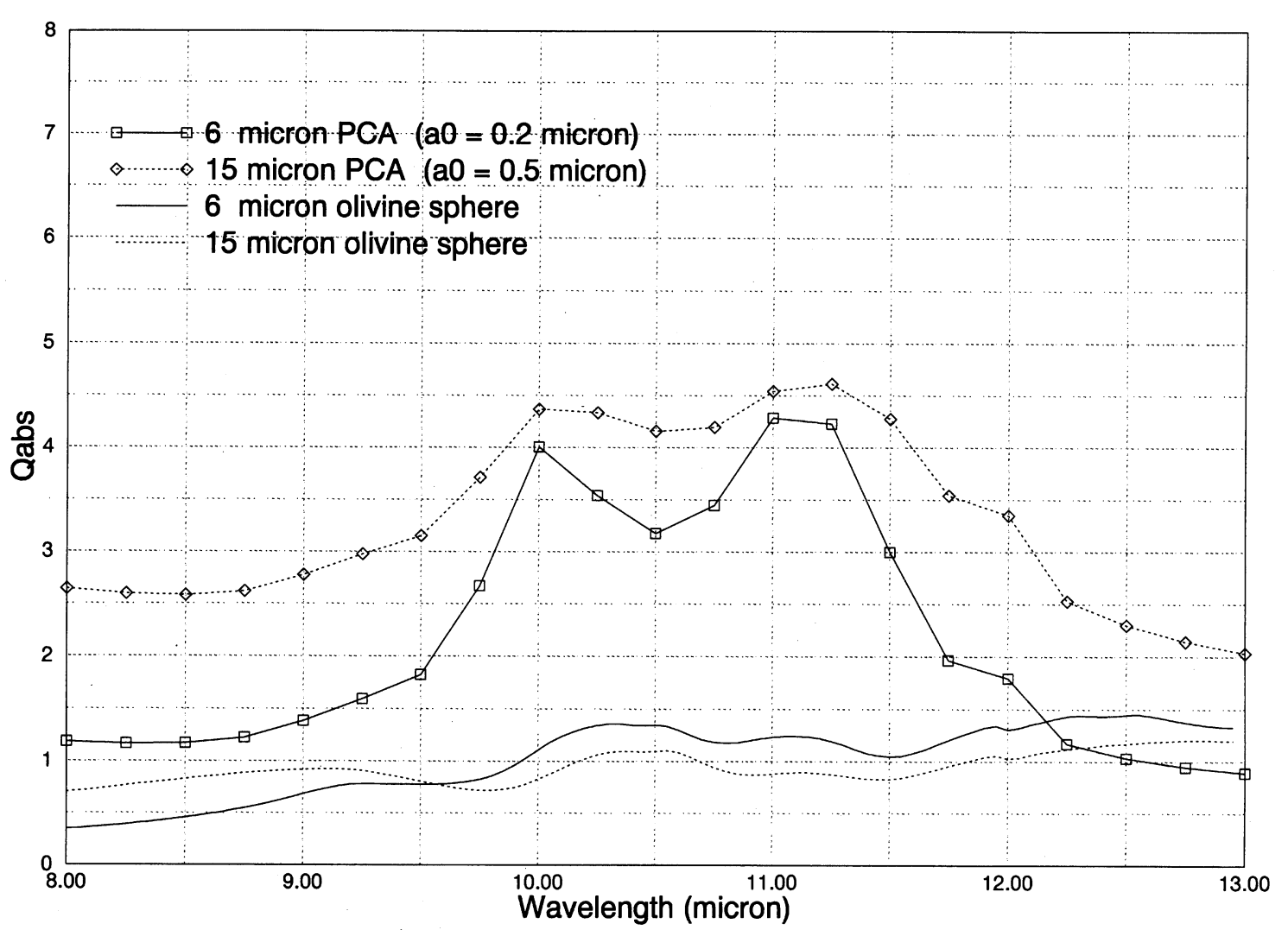

Fig. 6. Mid infrared absorption efficiencies of PCA with 27,000 monomers. Also shown are the absorption efficiencies for the volume-equivalent spheres.

lows. The scattering/absorption efficiency for PCA becomes constant as the size increase (Figs. 3 and 4). It means that the radiation pressure is proportional to the geometrical cross section. It is well known that the geometrical cross section of an aggregate with the fractal dimension $D$ is represented by $N^{1 / D}$ when $D>2$ and $N \gg 1$. The fractal dimension $D$ for PCA is nearly equal to 3 (Mukai et al., 1992) and the gravitational force are proportional to the mass $(\sim N)$. Then, the ratio of the radiation pressure force to gravitational force on PCA decreases as $N^{-1 / 3}$ and large PCA might stay on the stable orbit around $\beta$ Pic like spheres. Although the critical size would be larger than that for spheres, it seems plausible that the aggregates near the lower end of the size distribution, which is determined by the dynamical stability, dominate both the scattered light and thermal emission from the $\beta$ Pic disk.

We have modeled the dust particles around Beta Pictoris as the fluffy aggregates like chondritic porous IDPs and investigated the optical properties with Discrete Dipole Approximation with the a1-term method. The model can produce the neutral scattering and infrared silicate feature simultaneously in a wide size range. Furthermore, the observed high albedo and apparently high temperature result in a natural way. Our model, if eventually confirmed, lends support an implicit assumption in the previous studies that the same type of grains are responsible for the thermal infrared emission and the scattered light in visible. It should be emphasized that the model requires less adjustment of the dust size distribution and compositions in comparison with previous models based on spherical dust particles. This work is a first attempt to construct a self-consistent model of IDPs and dust particles in the $\beta$ Pic disk. In our future work, we plan to compare the phase functions and polarization of PCA computed by DDA and the a1-term method with the reference solutions provided by modal analysis (Okamoto, in this issue) to establish the range of applicability. Together with the infrared brightness distributions of the disk surface (Lagage and Pantin, 1994; Mouillet et al., 1997), our model is expected to provide important information on the spatial distribution of not only dust particles, but also of unseen planetesimals and planets in the disk.

Acknowledgments. We thank Dr. B. T. Draine for providing us with the original code of DDA. Helpful comments and criticisms from Dr. Y. Nakagawa and Dr. P. Artymowicz are greatly acknowledged.

\section{References}

Aitken, D. K., T. J. T. Moore, P. F. Roche, C. H. Smith, and C. M. Wright, Mid infrared spectroscopy of beta pictoris - constrains on the dust grain size-, Mon. Not. Astron. Roy. Soc., 265, L41-45, 1993.

Artymowicz, P., Radiation pressure forces on particles in the Beta Pictoris system, Astrophys. J., 335, L79-82, 1988.

Artymowicz, P., Beta Pictoris: An early solar system?, Annu. Rev. Earth Planet. Sci., 25, 175, 1997.

Artymowicz, P., C. Burrows, and F. Paresce, The structure of the Beta Pictoris disk and the properties of its particles, Astrophys. J., 337, 494513, 1989.

Backman, D. E., F. C. Whittebprn, and F. C. Gillet, Infrared observations and thermal models of the Beta Pictoris disk, Astrophys. J., 385, 670-679, 1992.

Draine, B. T., The discrete-dipole approximation and its application to in- 
terstellar graphite grains, Astrophys. J., 333, 848-872, 1988.

Draine, B. T. and H. M. Lee, Optical properties of interstellar graphite and silicate grains, Astrophys. J., 285, 89-108, 1984.

Greenberg, J. M. and A. Li, What are the true astronomical silicates?, Astron. Astrophys., 309, 258-266, 1996.

Kalas, P. and D. Jewitt, The detectability of Beta Pic-like circumstellar disks around nearby main sequence stars, Astron. J., 111, 1347-1355, 1996.

Knacke, R. F., S. B. Fajardo-acosta, C. M. Telesco, J. A. Hackwell, D. K. Lynch, and R. W. Russell, The silicates in the disk of beta Pictoris, Astrophys. J., 418, 440-454, 1993.

Lagage, P. O. and E. Pantin, Dust depletion in the inner disk of beta-pictoris as a possible indicator of planets, Nature, 369, 628-630, 1994.

Lecavelier des Etangs, A., G. Perrin, R. Ferlet, A. Vidal-Madjar, J. Lecacheux, M. Deleuil, and C. Gry, Observation of the central part of the beta-pictoris disk with an anti-blooming CCD, Astron. Astrophys., 274, 877-882, 1993.

Lecavelier des Etangs, A., A. Vidal-Madjar, and R. Ferelet, Dust distribution in disks supplied by small bodies: is the beta Pictoris disk a gigantic multi-cometary tail?, Astron. Astrophys., 307, 542-550, 1996.

Mathis, J. S., W. Rumpl, and K. H. Nordsieck, The size distribution of interstellar grains, Astrophys. J., 217, 425-433, 1977.

Matsuura, S., T. Matsumoto, H. Matsuhara, and M. Noda, Rocket-borne observations of the zodiacal light in the near-infrared wavelengths, Icarus, 115, 199-208, 1995.

Mouillet, D., A. M. Lagrange, J. L. Beuzit, and N. Renaud, A stellar coronograph for the COME-ON-PLUS adaptive opticssystem. II. First astronomical results, Astron. Astrophys., 324, 1083-1090, 1997.

Mukai, T. and C. Koike, Optical constants of olivine particles between wavelengths of 7 and 200 microns, Icarus, 87, 180-187, 1990.

Mukai, T., H. Ishimoto, T. Kozasa, J. Blum, and J. M. Greenberg, Radiation pressure forces of fluffy porous grains, Astron. Astrophys., 262, 315-320, 1992.

Nakano, T., Formation of planets around stars of various masses. III -
Massive and small-mass stars and the regions of planet formation, Mon Not. Astron. Roy. Soc., 235, 193-201, 1988.

Okamoto, H., Light scattering by clusters; the a1 term method, Opt. Rev., 2, 407-412, 1995.

Okamoto, H. and Y. Xu, Light scattering by irregular interplanetary dust particles, Earth Planets Space, 50, this issue, 577-585, 1998.

Ootsubo, T., T. Onaka, I. Yamamura, T. Tanabé, T. L. Roellig, K.-W. Chan, and T. Matsumoto, IRTS observation of the mid-infrared spectrum of the zodiacal emission, Earth Planets Space, 50, this issue, 507-511, 1998.

Paresce, F. and C. Burrows, Broad-band imaging of the Beta Pictoris circumstellar disk, Astrophys. J., 319, L23-25, 1987.

Reach, W. T., A. Abergel, F. Boulanger, F. Desert, M. Perault, J. P. Bernerd, J. Blommaert, C. Cesarsky, D. Cesarsky, L. Metcalfe, J. L. Puget, F. Sibille, and L. Vigroux, Mid-Infrared spectrum of the zodiacal light, Astron. Astrophys., 315, L381-384, 1996.

Sandford, S. A. and R. M. Walker, Laboratory infrared transmission spectra of individual interplanetary dust particles from 2.5 to 25 microns, Astrophys. J., 291, 838-851, 1985.

Telesco, C. M., R. Decher, E. E. Becklin, and R. D. Wolstencroft, Resolution of the circumstellar disk of Beta Pictoris at 10 and 20 microns, Nature, 335, 51-54, 1988.

Whitmire, D. P., J. Matese, and P. Whitman, Velocity streaming of IRAS main-sequence disk stars and the episodic enhancement of particulate disks by interstellar clouds, Astrophys. J., 388, 190-195, 1992.

Xing, Z. and M. S. Hanner, Light scattering by aggregate particles, Astron. Astrophys., 324, 805-820, 1997.

Yamamoto, S. and T. Mukai, Dust production by impacts of interstellar dust on Edgeworth-Kuiper Belt objects, Astron. Astrophys., 329, 785-791, 1998.

R. Nakamura (e-mail: ryosuke@kobe-u.ac.jp) 